BULL. AUSTRAL. MATH. SOC.

VOL. 8 (1973), 289-304.

\title{
Divergence of Fourier series
}

\section{Masako IzumI and Shin-ichi IzumI}

\author{
Carleson has proved that the Fourier series of functions \\ belonging to the class $L^{2}$ converge almost everywhere. \\ Improving his method, Hunt proved that the Fourier series of \\ functions belonging to the class $I^{p}(p>1)$ converge almost \\ everywhere. On the other hand, Kolmogoroft proved that there is \\ an integrable function whose Fourier series diverges almost \\ everywhere. We shall generalise Kolmogoroff's Theorem as \\ follows: There is a function belonging to the class $L(\log L)^{p}$ \\ $(p>0)$ whose Fourier series diverges almost everywhere. The \\ following problem is still open: whether "almost everywhere" in \\ the last theorem can be replaced by "everywhere" or not. This \\ problem is affirmatively answered for the class $L$ by \\ Kolmogoroff and for the class $L(\log \log L)^{p} \quad(0<p<1)$ by \\ Tandori.
}

\section{Introduction}

See [1], [3], [6], and [5] for the statements in the above abstract. Generalising Kolmogoroff's Theorem [6], Chen [2] and Prohorenko [4] proved that there is a function belonging to the class $L(\log \log L)^{P}(0<p<1)$ whose Fourier series diverges almost everywhere. We shall prove the following:

THEOREM. There is a function belonging to the class $L(\log L)^{p}$ $(p>0)$ whose Fourier series diverges almost everywhere.

For the proof of the theorem, we can suppose that $p$ is a positive 
integer since $L(\log L)^{p} \supset L(\log L)^{q}$ for $p<q$.

2.

We shall first prove the following

PROPOSITION. There are a sequence of trigonometric polynomials $\left(f_{n}\right)$ and a sequence of sets $\left(E_{n}\right)$ in the interval $(0,2 \pi)$, satisfying the following conditions:

$1^{\circ}$ the measure of $E_{n}$ tends to $2 \pi$ as $n \rightarrow \infty$,

$2^{\circ}$ for each $t \in E_{n}$, there corresponds a partial sum of $f_{n}$ whose absolute value is not less than $A \log n / \log \log n$.

We denote the mth Dirichlet kernel by $D_{m}(t)$ and the $m$ th Fejér kernel by $E_{m}(t)$; then

$$
D_{m}(t)=\frac{1}{2}+\sum_{k=1}^{m} \cos k t=\frac{\sin (m+1 / 2) t}{2 \sin t / 2}
$$

and

$$
\begin{aligned}
F_{m}(t) & =\frac{1}{m+1} \sum_{k=0}^{m} D_{k}(t)=\frac{\sin ^{2}(m+1) t}{(m+1)(2 \sin t / 2)^{2}} \\
& =\frac{1}{2}+\sum_{k=1}^{n}\left(1-\frac{k}{m+1}\right) \cos k t .
\end{aligned}
$$

It is known that

$$
0 \leq F_{m}(t) \leq A \min \left(m, 1 / m t^{2}\right) .
$$

By $F_{m}^{*}(t)$ we denote $F_{m}(t)$ without the constant term. Let $n$ be a large positive integer and take $n$ points $\left(c_{i}\right)$ on the interval $(0,2 \pi)$, defined by

$$
c_{i}=4 i \pi /(2 n+1) \quad(i=1,2, \ldots, n)
$$

and 


$$
c_{i}^{\prime}=\left(c_{i}+c_{i+1}\right) / 2 \quad(i=1,2, \ldots, n) .
$$

Let $\left(m_{i}\right)$ be an increasing sequence of integers defined by

$$
m_{i}=\frac{1}{2}\left((2 n+1)^{q_{3}} 3^{i}-1\right) \quad(i=1,2, \ldots, n)
$$

where $q$ is a positive integer $\geq p+2$. Then

$$
(2 n+1) \mid\left(2 m_{i}+1\right) \quad(i=1,2, \ldots, n) \text {. }
$$

Let $h$ be a function periodic with period $2 \pi$, defined by

$$
\begin{aligned}
h(t) & =0 \text { for } c_{i}^{-1 / n} \log \log n<t<c_{i}+1 / n \log \log n \quad(i=1,2, \ldots, n) \\
& =1 \text { otherwise in }(0,2 \pi) .
\end{aligned}
$$

We denote by $s_{m}(t, h)$ the $m$ th partial sum of the Fourier series of the function $h$ at the point $t$ and consider the trigonometric polynomial

$$
f_{n}(t)=\frac{1}{n} \sum_{i=1}^{n} F_{m_{i}}^{*}\left(t-c_{i}\right) s_{N}(t ; h)
$$

where $N=(2 n+1)^{p+2}$. The $\left(m_{j}+N\right)$ th partial sum of the Fourier series of $f_{n}$ is

$$
\begin{aligned}
s_{m_{j}+N}\left(t, f_{n}\right)=\frac{1}{n} \sum_{i=1}^{j} F_{m_{i}}^{*}\left(t-c_{i}\right) s_{N}(t ; h) & \\
& +\frac{1}{n} \sum_{i=j+1}^{n} \sum_{k=1}^{m_{j}}\left(1-\frac{k}{m_{i}+1}\right) \cos k\left(t-c_{i}\right) s_{N}(t ; h) \\
& +\frac{1}{n} \sum_{i=j+1}^{n} \sum_{k=m_{j}+1}^{m_{j+N}}\left(1-\frac{k}{m_{i}+1}\right) \cos k\left(t-c_{i}\right) s_{N+m_{j}-k}(t ; h) \\
= & P_{1}+P_{2}+P_{3} .
\end{aligned}
$$

We suppose that

$$
\log n<j<n-\sqrt{n}
$$

and

$$
c_{j}+2 / n \log \log n<t<c_{j}^{\prime}-1 / n \log \log n
$$


or

$$
c_{j}^{\prime+1 / n} \log \log n<t<c_{j+1}-2 / n \log \log n .
$$

Then we get

$$
\begin{aligned}
\left|P_{1}\right| & \leq \frac{A}{n} \sum_{i=1}^{j} \frac{1}{m_{i}} \frac{\sin ^{2}\left(m_{i}+1\right)\left(t-c_{i}\right)}{\left(2 \sin \left(t-c_{i}\right) / 2\right)^{2}}+A \\
& \leq \frac{A}{n} \sum_{i=1}^{j-1} \frac{n^{2}}{m_{i}(i-j)^{2}}+\frac{A}{n m_{j}}(n \log \log n)^{2}+A \leq A
\end{aligned}
$$

since $t-c_{i}>A(i-j) / n$ for $1 \leq i \leq j-1$, and similarly

$$
\begin{aligned}
P_{2}= & \frac{1}{n} \sum_{i=j+1}^{n} \frac{m_{j}^{+1}}{m_{i}+1} \sum_{k=1}^{m_{j}}\left(1-\frac{k}{m_{j}+1}\right) \cos k\left(t-c_{i}\right) s_{N}(t ; h) \\
& +\frac{1}{n} \sum_{i=j+1}^{n} \frac{m_{i}-m_{j}}{m_{i}+1} \sum_{k=1}^{m_{j}} \cos k\left(t-c_{i}\right) s_{N}(t ; h)
\end{aligned}
$$

where $t_{j}=t-c_{j}$. On the other hand, using Abel's transformation for the inner sumation of $P_{3}$, we write 


$$
\begin{aligned}
& P_{3}=\frac{1}{\pi n} \sum_{i=j+1}^{n} \sum_{k=m_{j}^{+1}}^{m_{j}^{+N}}\left(1-\frac{k}{m_{i}+1}\right) \cos k\left(t-c_{i}\right) \int_{0}^{2 \pi} h(u) \frac{\sin \left(N+m_{j}-k+1 / 2\right)(t-u)}{2 \sin (t-u) / 2} d u \\
&=\frac{1}{\pi n}\left[\sum_{i=j+1}^{n} \frac{1}{2}\left(1-\frac{m_{j}+N}{m_{i}+1}\right) \frac{\sin \left(m_{j}+N+1 / 2\right)\left(t-c_{i}\right)}{2 \sin \left(t-c_{i}\right) / 2} \int_{0}^{2 \pi} h(u) d u\right. \\
& \quad-\left(1-\frac{m_{j}^{+1}}{m_{i}+1}\right) \frac{\sin \left(m_{j}+1 / 2\right)\left(t-c_{i}\right)}{2 \sin \left(t-c_{i}\right) / 2} \int_{0}^{2 \pi} h(u) \frac{\sin (N-1 / 2)(t-u)}{2 \sin (t-u) / 2} d u \\
&+m_{j+N-1} \sum_{k=m_{j}+1} \frac{\sin (k+1 / 2)\left(t-c_{i}\right)}{2 \sin \left(t-c_{i}\right) / 2}\left\{\frac{1}{m_{i}+1} \int_{0}^{2 \pi} h(u) \frac{\sin \left(N+m_{j}-1 / 2\right)(t-u)}{2 \sin (t-u) / 2} d u\right. \\
&=Q_{1}-Q_{2}+\left(Q_{3}-Q_{4}+Q_{5}\right) ;
\end{aligned}
$$

then we can easily see that

$$
P_{2}^{\prime}-Q_{2}=O\left(\log n / n^{p+1}\right)
$$

and

$$
Q_{3}=O(1)
$$

Now we shall estimate $Q_{4}$.

$$
\begin{aligned}
Q_{4} & =\frac{1}{\pi n} \sum_{i=j+1}^{n} \frac{1}{2 \sin \left(t-c_{i}\right) / 2} \sum_{k=1}^{N-1} \sin \left(m_{j}+k+1 / 2\right)\left(t-c_{i}\right) \int_{0}^{2 \pi} h(u) \cos (N-k)(t-u) d u \\
& =\frac{1}{\pi n} \sum_{k=1}^{N-1} \sum_{i=j+1}^{n} \frac{\sin \left(m_{j}+k+1 / 2\right)\left(t-c_{i}\right)}{2 \sin \left(t-c_{i}\right) / 2} \int_{0}^{2} h(u) \cos (N-k)(t-u) d u,
\end{aligned}
$$

and we write the inner sum of $Q_{4}$ as follows: 


$$
\begin{aligned}
R_{k} & =\sum_{i=j+1}^{n} \frac{\sin \left(m_{j}+k+1 / 2\right)\left(t-c_{i}\right)}{2 \sin \left(t-c_{i}\right) / 2} \\
& =\sum_{i=j+1}^{n} \frac{\sin \left(m_{j}+k+1 / 2\right)\left(t_{j}-4(i-j) \pi /(2 n+1)\right)}{2 \sin \left(t_{j}-4(i-j) \pi /(2 n+1)\right) / 2} \\
& =\int_{j+1 / 2}^{n+1 / 2 \sin \left(m_{j}+k+1 / 2\right)\left(t_{j}-4(v-j) \pi /(2 n+1)\right)} \\
& =R_{k}^{\prime}+R_{k}^{\prime \prime},
\end{aligned}
$$

where

$$
J(v)=[v]-v+1 / 2=\sum_{l=1}^{\infty} \frac{\sin 2 \pi l v}{2 \pi l}
$$

and further

$$
\begin{aligned}
R_{k}^{\prime} & =\frac{2 n+1}{4 \pi} \int_{-2+4 j \pi /(2 n+1)+t_{j}}^{t-2 \pi /(2 n+1)} \sin \left(m_{j}+k+1 / 2\right) w\left(\frac{1}{w}-\frac{2 \sin w / 2-w}{2 \omega \sin w / 2}\right) d w \\
& =S_{k}^{\prime}+S_{k}^{\prime \prime} .
\end{aligned}
$$

If $t$ belongs to the interval (1), then

$$
t_{j}-2 \pi /(2 n+1) \leq-1 / n \log \log n
$$

and then $S_{k}^{\prime}=0\left(n^{2} \log \log n / m_{j}\right)$. Similarly $S_{k}^{\prime \prime}$ is also of order not greater than $n^{3} \log \log n / m_{j} \log n$, by using the second mean value theorem and the fact that $(w / 2 \sin w / 2)-1>0$, and decreasing in the interval of integration. Therefore

$$
Q_{4}^{\prime}=\frac{1}{\pi n} \sum_{k=1}^{N-1} R_{k}^{\prime} \int_{0}^{2 \pi} h(u) \cos (N-k)(t-u) d u=O(1) .
$$

On the other hand, if $t$ belongs to the interval (2), then

$$
t_{j}-2 \pi /(2 n+1) \geq 1 / n \log \log n
$$

and we can estimate 


$$
\begin{aligned}
& S_{k}^{\prime}=\frac{2 n+1}{4 \pi}\left\{\int_{-\infty}^{\infty} \frac{\sin w}{w} d w-\right. \\
& \left.-\left\{\int_{t_{j}-2 \pi /(2 n+1)}^{\infty}+\int_{-\infty}^{-2 \pi+4 j \pi /(2 n+1)+t} j\right) \frac{\sin \left(m_{j}+k+1 / 2\right) \omega}{\omega} d \omega\right\} \\
& =A(2 n+1)+o\left(n^{2} \log \log n / m_{j}\right) \text {, } \\
& S_{k}^{\prime \prime}=A \frac{2 n+1}{4 \pi}\left\{\frac{n}{\log n} \int_{-2 \pi+4 j \pi /(2 n+1)+t_{j}}^{-1 / n} \frac{\sin \left(m_{j}+k+1 / 2\right) \omega}{w} d v\right. \\
& \left.+\int_{-1 / n}^{t} j^{-2 \pi /(2 n+1)} \sin \left(m_{j}+k+1 / 2\right) w\left(\frac{w-2 \sin w / 2}{2 \sin w / 2}\right) d \omega\right\} \\
& =O\left(n^{3} \log \log n / m_{j} \log n\right)+o\left(1 / n^{2}\right),
\end{aligned}
$$

similarly as before; so that $Q_{4}^{\prime}$ is also bounded for $t$ in the interval (2). Hence we have to estimate the rest of $Q_{4}$ :

$$
\begin{aligned}
Q_{4}^{\prime \prime} & =Q_{4}-Q_{4}^{\prime} \\
& =\frac{1}{\pi n} \sum_{k=1}^{N-1} R_{k}^{\prime \prime} \int_{0}^{2} h(u) \cos (N-k)(t-u) d u \\
& =\frac{1}{\pi n} \sum_{k=1}^{N-1} R_{n}^{\prime \prime}\left(s_{N-k}(t ; h)-s_{N-k-1}(t ; h)\right) \\
& =\frac{1}{\pi n}\left\{-s_{0}(t ; h) R_{N-1}^{\prime \prime}+s_{N-1}(t ; h) R_{1}^{\prime \prime}+\sum_{k=1}^{N-2} s_{k}(t ; h)\left(R_{N-k^{\prime \prime}}^{\prime \prime} R_{N-k-1}^{\prime \prime}\right)\right\} \\
& =-T_{1}+T_{2}+T_{3},
\end{aligned}
$$

where 


$$
\begin{aligned}
& R_{k}^{\prime \prime}=-\int_{j+1 / 2}^{n+1 / 2} J(v) \frac{d}{d v}\left(\frac{\sin \left(m_{j}+k+1 / 2\right)\left(t_{j}-4(v-j) \pi /(2 n+1)\right)}{2 \sin \left(t_{j}-4(v-j) \pi /(2 n+1)\right) / 2}\right) d v \\
& =\frac{-1}{2 \pi} \sum_{i=1}^{\infty} \frac{1}{\tau} \int_{j+1 / 2}^{n+1 / 2} \sin 2 \pi \tau_{v} \frac{d}{d v}\left(\frac{\sin \left(m_{j}+k+1 / 2\right)\left(t_{j}-4(v-j) \pi /(2 n+1)\right)}{2 \sin \left(t_{j}-4(v-j) \pi /(2 n+1)\right) / 2}\right) d v \\
& =\sum_{i=1}^{\infty} \int_{j+1 / 2}^{n+1 / 2} \frac{\cos 2 \pi l v \cdot \sin \left(m_{j}+k+1 / 2\right)\left(t j^{-4(v-j) \pi /(2 n+1))}\right.}{2 \sin \left(t_{j}-4(v-j) \pi /(2 n+1)\right) / 2} d v \\
& =\frac{n+1 / 2}{2 \pi} \sum_{i=1}^{\infty} \int_{-2+4 j \pi /(2 n+1)+t_{j}}^{t_{j}-2 \pi /(2 n+1)} \frac{\cos 2(n+1 / 2)\left(\omega-t_{j}\right) \sin \left(m_{j}+k+1 / 2\right) \omega}{2 \sin w / 2} d \omega \\
& =\frac{n+1 / 2}{2 \pi} \int_{1 / 2}^{\infty}(d l+d J(l)) \int_{-2+4 j \pi /(2 n+1)+t_{j}}^{t} \\
& \cdot \frac{\cos 2(m+1 / 2)\left(w-t_{j}\right) \sin \left(m_{j}+k+1 / 2\right) w}{2 \sin w / 2} d v \\
& =v_{k}^{\prime}+v_{k}^{\prime \prime} \text {. }
\end{aligned}
$$

We shall first estimate $T_{1}$. Changing the order of integration and using the second mean value theorem, the first part of $R_{N-1}^{\prime \prime}$ becomes

$$
\begin{aligned}
& \frac{1}{n} U_{n-1}^{\prime}=\frac{n+1 / 2}{n} \int_{-2 \pi+4 j \pi /(2 n+1)+t_{j}}^{t} \frac{\sin \left(m_{j}+N-1 / 2\right) \omega}{2 \sin w / 2} d v . \\
& \text { - } \int_{1 / 2}^{\infty} \cos \tau(n+1 / 2)\left(\omega-t_{j}\right) d \tau \\
& =\frac{1}{n} \lim _{M \rightarrow \infty} \int_{-2 \pi+4 j \pi /(2 n+1)+t_{j}}^{t,-2 \pi /(2 n+1)} \frac{\sin M(n+1 / 2)\left(\omega-\dot{t}_{j}\right) \cdot \sin \left(m_{j}+N-1 / 2\right) \omega}{\left(\omega-t_{j}\right) \cdot 2 \sin w / 2} d \omega \\
& -\frac{1}{n} \int_{-2 \pi+4 j \pi /(2 n+1)+t_{j}}^{t} \frac{\sin (n+1 / 2)\left(w-t_{j}\right) / 2 \cdot \sin \left(m_{j}+N-1 / 2\right) w}{\left(w-t_{j}\right) \cdot 2 \sin w / 2} d w \\
& =O(1) \text {, }
\end{aligned}
$$

and the second part of $R_{N-1}^{\prime \prime}$ is 


$$
\begin{aligned}
& \frac{1}{n} U_{N-1}^{\prime \prime}=\frac{n+1 / 2}{n} \int_{1 / 2}^{\infty} d J(Z) \int_{-2 \pi+4 j \pi /(2 n+1)+t_{j}}^{t} \cdot \\
& \text { - } \frac{\cos 2(n+1 / 2)\left(\omega-t_{j}\right) \cdot \sin \left(m_{j}+N-1 / 2\right) \omega}{2 \sin \omega / 2} d \omega \\
& =\frac{(n+1 / 2)^{2}}{n} \int_{I / 2} J(I) d l \int_{-2 \pi+4 j \pi /(2 n+1)+t_{j}}^{t_{j}-2 \pi /(2 n+1)} \\
& \text { - } \frac{\left(w-t_{j}\right) \sin 2(n+1 / 2)\left(w-t_{j}\right) \cdot \sin \left(m_{j}+N-1 / 2\right) w}{2 \sin w / 2} d v \\
& =\frac{(n+1 / 2)^{2}}{n} \lim _{M \rightarrow \infty} \int_{-2 \pi+4 j \pi /(2 n+1)+t_{j}}^{t_{j}-2 \pi /(2 n+1)} \frac{\left(w-t_{j}\right) \sin \left(m_{j}+N-1 / 2\right) w}{2 \sin w / 2} d \omega \\
& \cdot \int_{1 / 2}^{M} J(Z) \sin Z(n+1 / 2)\left(w-t_{j}\right) d z
\end{aligned}
$$

where we can suppose that $M$ is an even integer. The last inner integral is

$$
\begin{aligned}
& \int_{1 / 2}^{M} J(z) \sin (n+1 / 2)\left(w-t_{j}\right) d z \\
& =\sum_{k=1}^{\infty} \frac{1}{2 \pi k} \int_{1 / 2}^{M} \sin 2 l k \pi \cdot \sin (n+1 / 2)\left(\omega-t_{j}\right) d l \\
& =\sum_{k=1}^{\infty} \frac{1}{4 \pi k} \int_{1 / 2}^{M}\left\{\cos (n+1 / 2)\left(\omega-t_{j}-2 \pi k\right) z-\cos \left((n+1 / 2)\left(\omega-t_{j}\right)+2 \pi k\right) z\right\} d z \\
& \therefore \frac{1}{4 \pi} \sum_{k=1} \frac{1}{k}\left\{\frac{\sin \left((n+1 / 2)\left(\omega-t_{j}\right) / 2-k \pi\right)}{(n+1 / 2)\left(w-t_{j}\right)-2 k \pi}-\frac{\sin \left((n+1 / 2)\left(\omega-t_{j}\right) / 2+k \pi\right)}{(n+1 / 2)\left(\omega-t_{j}\right)+2 k \pi}\right\} \\
& +\frac{1}{4 \pi} \sum_{k=1}^{\infty} \frac{1}{k}\left\{\frac{\sin \left((n+1 / 2)\left(\omega-t_{j}\right)-2 k \pi\right) M}{(n+1 / 2)\left(w-t_{j}\right)-2 k \pi}-\frac{\sin \left((n+1 / 2)\left(\omega-t_{j}\right)+2 k \pi\right) M}{(n+1 / 2)\left(\omega-t_{j}\right)+2 k \pi}\right\} \\
& =-\sin (n+1 / 2)\left(\omega-t_{j}\right) / 2 \sum_{k=1}^{\infty} \frac{(-1)^{k}}{(n+1 / 2)^{2}\left(\omega-t_{j}\right)^{2}-(2 k \pi)^{2}} \\
& +\sum_{k=1}^{\infty} \frac{\sin \left((n+1 / 2)\left(w-t_{j}\right)-2 k \pi\right) M}{\left[(n+1 / 2)\left(w-t_{j}\right)-2 k \pi\left[(n+1 / 2)\left(w-t_{j}\right)+2 k \pi\right\}\right.},
\end{aligned}
$$

where we use the convention that $\sin x / x=1$ for $x=0$. Substituting 
the first term on the right side into $U_{n-1}^{\prime \prime} / n$, we get

$$
\begin{aligned}
& -\frac{(n+1 / 2)^{2}}{n} \int_{t_{j}-2 \pi+4 j \pi /(2 n+1)}^{t_{j}-2 \pi /(2 n+1)} \frac{\left(w-t_{j}\right) \sin \left(m_{j}+N-1 / 2\right) w}{2 \sin w / 2} \cdot \\
& \cdot \sin (n+1 / 2)\left(w-t_{j}\right) / 2 \sum_{k=1}^{\infty} \frac{(-1)^{k}}{(n+1 / 2)^{2}\left(w-t_{j}\right)^{2}-(2 k \pi)^{2}} d \omega \\
& =-\frac{(n+1 / 2)^{2}}{n} \sum_{m=1}^{n-j} \int_{t_{j}-2(2 m+1) \pi /(2 n+1)}^{t_{j}-2(2 m-1) \pi /(2 n+1)} D_{m_{j}+N-1}^{(w) \cdot} \\
& \quad \cdot\left(w-t_{j}\right) \sum_{k=1}^{\infty} \frac{(-1)^{k} \sin (n+1 / 2)\left(w-t_{j}\right) \cdot / 2}{(n+1 / 2)^{2}\left(w-t_{j}\right)^{2}-(2 k \pi)^{2}} d \omega \\
& =0(1)
\end{aligned}
$$

by the second mean value theorem, since the last sum is expressed as the difference of bounded monotone increasing functions in each interval of integration. Therefore

$$
\begin{aligned}
& \frac{1}{n} U_{N-1}^{\prime \prime}=\frac{1}{n} \lim _{M \rightarrow \infty} \sum_{k=1}^{\infty} \int_{t_{j}-2 \pi+4 j \pi /(2 n+1)}^{t j^{-2 \pi /(2 n+1)}} \frac{\left(w-t_{j}\right) \sin \left(m_{j}+N-1 / 2\right) w}{2 \sin w / 2\left(w-\left(t_{j}+2 k \pi /(n+1 / 2)\right] / 2\right)} \\
&=\frac{\sin \left(w-\left(t_{j}-2 k \pi /(n+1 / 2)\right)(n+1 / 2)\right) M}{w-\left(t_{j}-2 k \pi /(n+1 / 2)\right)} d w+o(1) \\
&=\frac{\pi}{2 n} \sum_{k=1}^{n-j} \frac{\sin \left(m_{j}+N-1 / 2\right)\left(t_{j}-2 k \pi /(n+1 / 2)\right)}{2 \sin \left(t_{j}-2 k \pi /(n+1 / 2)\right) / 2}+o(1) \\
& \sum_{i=j+1}^{n} \frac{\sin \left(m_{j}+N+1 / 2\right)\left(t-c_{i}\right)}{2 \sin \left(t-c_{i}\right) / 2}+o(1)
\end{aligned}
$$

by the Dirichlet formula and then we get

$$
\begin{aligned}
T_{1} & =\frac{1}{2 n} \boldsymbol{s}_{0}(t ; h) \sum_{i=j+1}^{n} \frac{\sin \left(m_{j}+N+1 / 2\right)\left(t-c_{i}\right)}{2 \sin \left(t-c_{i}\right) / 2}+O(1) \\
& =Q_{1}+O(\log \log n) .
\end{aligned}
$$

By a similar estimation, we can see that 


$$
T_{2}=\frac{1}{2 n} s_{N-1}(t ; h) \sum_{i=j+1}^{n} \frac{\sin \left(m_{j}+3 / 2\right)\left(t-c_{i}\right)}{2 \sin \left(t-c_{i}\right) / 2}+O(1)
$$

Now

$$
T_{3}=\frac{1}{\pi n} \sum_{k=1}^{N-2} s_{k}(t ; h) \int_{j+1 / 2}^{n+1 / 2} J(v) \frac{d}{d v} \sin \left(m_{j}+k\right)(t-4 v \pi /(2 n+1)) d v
$$

where the above integral equals

$$
T_{3}^{\prime}=\frac{4 \pi\left(m_{j}+k\right)}{2 n+1} \int_{j+1 / 2}^{n+1 / 2} J(v) \cos \left(m_{j}+k\right)(t-4 v \pi /(2 n+1)) d v .
$$

By the transformation $4 v \pi /(2 n+1)=w$,

$T_{3}^{\prime}$

$=\left(m_{j}+k\right) \int_{2(2 j+1) \pi /(2 n+1)}^{2 \pi} J\left(\frac{(2 n+1) w}{4}\right) \cos \left(m_{j}+k\right)(t-w) d w$

$=\frac{m_{j}+k}{2} \sum_{i=1}^{\infty} \frac{1}{2} \int_{2(2 j+1) \pi /(2 n+1)}^{2 \pi} \sin (n+1 / 2) \tau w \cdot \cos \left(m_{j}+k\right)(t-w) d w$

$=-\frac{m_{j}+k}{4 \pi} \sum_{l=1}^{\infty} \frac{1}{l}\left\{\frac{\cos \left(\left\{(n+1 / 2) z-\left(m_{j}+k\right)\right\} w+\left(m_{j}+k\right) t\right)}{(n+1 / 2) z\left(m_{j}+k\right)}\right.$

$\left.\left.+\frac{\cos \left(\left\{(n+1 / 2) Z+\left(m_{j}+k\right)\right\} \omega-\left(m_{j}+k\right) t\right)}{(n+1 / 2) Z+\left(m_{j}+k\right)}\right]_{2(2 j+1) \pi /(2 n+1)}^{2 \pi}\right\}$

$=-\frac{m_{j}+k}{4 \pi} \sum_{i=1}^{\infty} \frac{1}{2}\left\{\frac{(-1)^{2} \cos \left(m_{j}+k\right) t}{(n+1 / 2) 2-\left(m_{j}+k\right)}-\frac{(-1)^{2} \cos \left(\left(m_{j}+k\right)(2 j+1) \pi /(n+1 / 2)+\left(m_{j}+k\right) t\right)}{(n+1 / 2) Z-\left(m_{j}+k\right)}\right.$

$$
+\frac{(-1)^{2} \cos \left(m_{j}+k\right) t}{(n+1 / 2) \tau+\left(m_{j}+k\right)}-\frac{(-1)^{2} \cos \left(\left(m_{j}+k\right)(2 j+1) \pi /(n+1 / 2)-\left(m_{j}+k\right) t\right)}{(n+1 / 2) \tau+\left(m_{j}+k\right)}
$$

$=-\frac{m_{j}^{+k}}{4 \pi}\left[\left\{\cos \left(m_{j}+k\right) t-\cos \left(\left(m_{j}+k\right)((2 j+1) \pi /(n+1 / 2)+t)\right)\right\} \sum_{l=1}^{\infty} \frac{(-1)^{2}}{2\left\{(n+1 / 2) l-\left(m_{j}+k\right)\right\}}\right.$

$\left.+\left\{\cos \left(m_{j}+k\right) t-\cos \left(\left(m_{j}+k\right)((2 j+1) \pi /(n+1 / 2)-t)\right)\right] \sum_{l=1}^{\infty} \frac{(-1)^{2}}{2\left\{(n+1 / 2) \imath+\left(m_{j}+k\right)\right\}}\right]$

$=A\left\{\cos \left(m_{j}+k\right) t-\cos \left(m_{j}+k\right)(t+(2 j+1) \pi /(n+1 / 2))\right\}$

$$
+A\left\{\cos \left(m_{j}+k\right) t-\cos \left(m_{j}+k\right)(t-(2 j+1) \pi /(n+1 / 2))\right\}+o\left[n^{2} / m_{j}\right),
$$

where * means the sum omitting the term with vanishing denominator. 


\section{Therefore}

$$
\begin{aligned}
T_{3} & =\frac{1}{n} \sum_{k=1}^{N-2} s_{k}(t ; h)\left\{A \cos \left(m_{j}+k\right) t-A\left(m_{j}+k\right)(t+(2 j+1) \pi /(n+2))\right. \\
& =V_{1}-V_{2}-V_{3}+O(1),
\end{aligned}
$$

where

$$
\begin{aligned}
& V_{1}=\frac{A}{n} \int_{0}^{2 \pi} h(t-u) \sum_{k=1}^{N-2} D_{k}(u) \cos \left(m_{j}+k\right) t d u \\
& =\frac{A}{n} \sum_{k=1}^{N-2} \cos \left(m_{j}+k\right) t \\
& -\frac{A}{n} \int_{0}^{2 \pi}(1-h(t-u))\left\{\frac{\cos \left((u-t) / 2+u / 2-m_{j} t\right)-\cos \left((N-3 / 2)(u-t)+u / 2-m_{j} t\right)}{2 \sin u / 2 \cdot 2 \sin (u-t) / 2}\right. \\
& =o\left(\frac{1}{j}\right)+o\left(\frac{1}{n} \sum_{i=1}^{n} \int_{t-c_{i}-1 / n \log \log n}^{t-c_{i}+1 / n \log \log n} \frac{d u}{\sin u / 2 \cdot \sin (u-t) / 2}\right) \\
& =O(1)+o\left(\frac{1}{n} \sum_{\substack{i=1 \\
i \neq j}}^{n} \frac{n^{2}}{i-j \mid i n \log \log n}+\frac{1}{n} \frac{(n \log \log n)^{2}}{n \log \log n}\right) \\
& =O(\log \log n)
\end{aligned}
$$$$
\left.+\frac{\cos \left((u+t) / 2+u / 2+m_{j} t\right)-\cos \left((N-3 / 2)(u+t)+u / 2+m_{j} t\right)}{2 \sin u / 2 \cdot 2 \sin (u+t) / 2}\right\} d u
$$

and similarly $V_{2}$ and $V_{3}$ are also $O(\log \log n)$. Collecting the above estimates, we get

$$
\begin{aligned}
8_{m_{j}+N}\left(t ; f_{n}\right) & =Q_{1}-Q_{4}+O(\log \log n)=T_{2}+O(\log \log n) \\
& =\frac{\sin \left(m_{j}+1 / 2\right) t_{i}}{n} \sum_{i=j+1}^{n} \frac{1}{2 \sin \left(t-c_{i}\right) / 2}+O(\log \log n)
\end{aligned}
$$

for all $t$ belonging to the intervals (1) and (2).

We denote by $E_{n, j}$ the set of $t$ in the intervals (1) and (2), satisfying the condition 


$$
\begin{aligned}
& \text { Divergence of Fourier series } \\
& \left|\sin \left(m_{j}+1 / 2\right) t_{j}\right|>1 / \log \log n
\end{aligned}
$$

then

$$
\left|s_{m_{j}+N}\left(t ; f_{n}\right)\right| \geq A \log n / \log \log n \text { for all } t \text { in } E_{n, j} .
$$

We write

$$
E_{n}=\bigvee\left(E_{n, j} ; \log n<j<n-\sqrt{n}\right) ;
$$

then $\left(f_{n}\right)$ and $\left(E_{n}\right)$ satisfy the conditions of the proposition stated at the beginning of this section.

3.

Let $\left(r_{k}(x)\right)$ be the Rademacher system on the interval $(0,1)$ and we shall consider the function

$$
f(t, x)=\sum_{k=1}^{\infty} f_{p_{k}}\left(q_{k} t\right) r_{k}(x) / \log \log p_{k}
$$

where $f_{p_{k}}$ is defined in Section 2 and $\left(q_{k}\right)$ is the sequence of integers defined such that $f_{p_{k}}\left(q_{k+1} t\right)$ have no overlapping terms for each $k$ and $\left(p_{k}\right)$ will be determined such that $f \in L(\log L)^{p}$. We write

$$
Y(t)=t(\log (t+e))^{p}, Z(t)=Y(\sqrt{t}) \text { for } t>0 ;
$$

then, by the Jensen inequalities, we get (cf. [5]), 
302

Masako Izumi and Shin-ichi Izumi

$$
\begin{aligned}
\int_{0}^{1} d x \int_{0}^{2 \pi} Y(|f(t, x)|) d t & =\int_{0}^{2 \pi} d t \int_{0}^{1} Y(|f(t ; x)|) d x \\
& =\int_{0}^{2 \pi} d t \int_{0}^{1} Z\left(|f(t, x)|^{2}\right) d x \\
& \leq \int_{0}^{2 \pi} z\left(\int_{0}^{1}(f(t, x))^{2} d x\right) d t \\
& =\int_{0}^{2 \pi} z\left(\sum_{k=1}^{\infty}\left(f_{p_{k}}\left(q_{k} t\right) / \log \log p_{k}\right)^{2}\right) d t \\
& \left.\leq \int_{0}^{2 \pi} \sum_{k=1}^{\infty} z\left(\mid f_{p_{k}}\left(q_{k} t\right) / \log \log p_{k}\right)^{2}\right) d t \\
& =\int_{0}^{2 \pi} \sum_{k=1}^{\infty} Y\left(f_{p_{k}}\left(q_{k} t\right) / \log \log p_{k}\right) d t \\
& =\sum_{k=1}^{\infty} \int_{0}^{2 \pi} Y\left(f_{p_{k}}\left(q_{k} t\right) / \log \log p_{k}\right) d t .
\end{aligned}
$$

If the last series is convergent, then there is an $x_{0}$ such that $f\left(t, x_{0}\right) \in L(\log L)^{p}$. Therefore it is sufficient to prove that

$$
\int_{0}^{2 \pi} y\left(\left|f_{n}(t)\right| / \log \log n\right) d t \rightarrow 0 \text { as } n \rightarrow \infty
$$

which follows from

$$
\int_{0}^{2 \pi} y\left(\left|f_{n}(t)\right|\right) d t \leq A \text { for all } n
$$

The left side integral is

$$
\begin{aligned}
& \frac{1}{n}\left(\int_{0}^{2 \pi /(2 n+1)}+\sum_{k=1}^{n-1} \int_{c_{k}-2 \pi /(2 n+1)}^{c_{k}+2 \pi /(2 n+1)}+\int_{c_{n-1}+2 \pi /(2 n+1)}^{2 \pi}\right) \\
& \quad\left(\sum_{i=1}^{n} F_{m_{i}^{*}}^{*}\left(t-c_{i}\right) s_{N}(t ; h)\right)\left|\log \left(\left|f_{n}(t)\right|+e\right)\right|^{p} d t \\
& =\frac{1}{n}\left(w_{0}+\sum_{k=1}^{n-1} w_{k}+w_{n}\right) .
\end{aligned}
$$

https://doi.org/10.1017/S0004972700042532 Published online by Cambridge University Press 
We shall prove that each $W_{k}$ is bounded. For $1 \leq k \leq n-1$, we write

$$
\begin{aligned}
W_{k} & =\int_{c_{k}-2 \pi /(2 n+1)}^{c_{k}+2 \pi /(2 n+1)}=\int_{c_{k}-2 \pi /(2 n+1)}^{c_{k}}+\int_{c_{k}}^{c_{k}+2 \pi /(2 n+1)} \\
& =W_{k}^{\prime}+W_{k}^{\prime \prime}
\end{aligned}
$$

and

$$
\begin{aligned}
w_{k}^{\prime \prime} & =\int_{c_{k}}^{c_{k}+1 / m_{k}}+\int_{c_{k}+1 / m_{k}}^{c_{k}+1 / 2 n \log \log n}+\int_{c_{k}+1 / 2 n \log \log n}^{c_{k}+2 \pi /(2 n+1)} \\
& =x_{1}+x_{2}+x_{3} .
\end{aligned}
$$

Since

$$
\log \left(\left|f_{n}(t)\right|+e\right) \leq A n
$$

and

$$
{ }_{s_{N}}(t ; h)=O(n \log \log n / N) \text { for } c_{k}<t<c_{k}+1 / 2 n \log \log n \text {, }
$$

we have

$$
\begin{aligned}
\left|x_{1}\right| & \leq \frac{A n^{p+1} \log \log n}{n} \int_{c_{k}}^{c_{k}^{+1 / m} k}\left(F_{m_{k}}\left(t-c_{k}\right)+\sum_{\substack{i=1 \\
i \neq k}}^{n} F_{m_{i}}\left(t-c_{i}\right)\right) d t+A \\
& \leq \frac{A n^{p+1} \log \log n}{n}\left[A+\sum_{\substack{i=1 \\
i \neq k}}^{n} \frac{A n^{2}}{m_{i}(i-k)^{2}} \frac{1}{m_{k}}\right)^{+A} \\
& \leq A, \\
\left|X_{2}\right| & \leq \frac{A r^{p+1} \log \log n}{n}\left(\int_{1 / m_{k}}^{1 / 2 n \log \log n} \frac{d t}{m_{k} t^{2}}+A\right) \\
& \leq A
\end{aligned}
$$

and

$$
\begin{aligned}
X_{3} & \leq A n^{p}\left(\int_{1 / 2 n \log \log n}^{2 \pi /(2 n+1)}\left(\frac{1}{m_{k} t^{2}}+\sum_{\substack{i=1 \\
i \neq k}}^{n} \frac{n^{2}}{m_{i}(i-k)^{2}}\right) d t\right) \\
& \leq A .
\end{aligned}
$$


Therefore we have proved that $W_{k}^{\prime \prime}$ is bounded. Similarly $W_{k}^{\prime}$ is bounded. Since $W_{0}$ and $W_{n}$ are also bounded, all $W_{k}$ are bounded, which proves (3).

Thus we have proved that $f\left(t, x_{0}\right) \in L(\log L)^{p}$. The theorem is now completely proved, since the Fourier series of $f\left(t, x_{0}\right)$ diverges almost everywhere which follows from Section 2.

\section{References}

[1] Lennart Carleson, "On convergence and growth of partial sums of Fourier series", Acta Math. 116 (1966).

[2] Yung-ming Chen, "On Kolmogoroff's divergent Fourier series", Arch. Math. 14 (1963), 116-119.

[3] Richard A. Hunt, "On the convergence of Fourier series", Orthogonal expansions and their contimuous analogues, 235-255 (Proceedings Conf. Edwardsville, Illinois, 1967. Southern Illinois Univ. Press, Carbondale, Illinois, 1968); (cf. MR38\#6296).

[4] В.Н. Прохоренно [V.І. Prohorenko], "О расходящнхся рядах фурье" [Divergent Fourier series], Mat. Sb. (N.S.) 75 (117) (1968), 185-198.

[5] Károly Tandori, "Ein Divergenzsatz für Fourierreihen", Acta Sci. Math. 30 (1969), 43-48.

[6] A. Zygmund, Trigonometric series, Vol. 1, 2nd ed. (Cambridge University Press, Cambridge, 1959; reprinted 1968).

Department of Mathematics, Institute of Advanced Studies, Australian National University, Canberra, ACT. 